\title{
Volume yield and tree species diversity of three protected areas in Akure forest reserve, Ondo State, Nigeria
}

\author{
R. I. Adeseko, A. S. Akinbowale*, C. I. Arinzechi and A. A. Adeboyejo \\ Department of Forestry and Wood Technology, The Federal University of Technology, Akure, Ondo State, Nigeria \\ *Corresponding Author: akinbowaleas@futa.edu.ng
}

\begin{abstract}
The roles of protected areas in biodiversity conservation were assessed in this study. This was achieved by assessing tree species diversity and volume yield of three protected areas in Akure Forest Reserve, Ondo State, Nigeria. Two sampling plots of 50 $\mathrm{m} \times 50 \mathrm{~m}$ were laid in each of the sites using systematic line transect. Trees with DBH $>10 \mathrm{~cm}$ were identified in each plot, their frequency of occurrence were ascertained and categorized into families. All tree growth variables were measured in each of the study sites. The results obtained from this study indicated that the three selected protected areas within the forest reserve are rich in trees species. A total of 264 stems $^{-1}{ }^{-1}$ were observed in the SNR, 198 stems ha $^{-1}$ were recorded in the Buffer zone and Enrichment

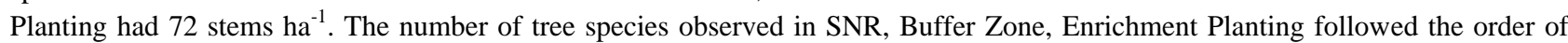
37> $31>25$. Shannon Weiner index of 2.97, 3.10 and 3.00 were obtained in the buffer zone, SNR and Enrichment planting site respectively. SNR had the highest volume of $461.74 \mathrm{~m}^{3} \mathrm{ha}^{-1}$, this was followed by the Buffer zone with $424.46 \mathrm{~m}^{3} \mathrm{ha}^{-1}$ and the Enrichment planting $\left(138.28 \mathrm{~m}^{3} \mathrm{ha}^{-1}\right)$. The high biodiversity indices are indication that biodiversity can be conserved through in situ method if proper managerial actions are put in place. The study therefore recommended that the remaining protected areas should be safeguarded from anthropogenic activities and more protected areas be established.
\end{abstract}

Keywords: Protected area, Conservation, Biodiversity, Volume.

\section{INTRODUCTION}

Forest biodiversity protection relies on the ability to assess hot spots, quantify and predict spatial and temporal trends of key species, maintain a natural disturbance regime and limit harmful human activities (Stohlgren et al., 1999). They are geographical space, recognized, dedicated and managed, through legal or other effective means, to achieve the long-term conservation of nature with associated ecosystem services and cultural values (Dudley, 2008).

Protected areas was defined by the International Union for Conservation of Nature (IUCN) as an area of land and/or sea especially dedicated to the protection and maintenance of biological diversity, and of natural and associated cultural resources, and managed through legal or other effective means (IUCN, 1994). Forest protected areas help conserve ecosystems that provide habitat, shelter, food, raw materials, genetic materials, a barrier against disasters, a stable source of resources and many other ecosystem goods and services and thus can have an important role in helping species, people and countries adapt to climate change. They can thus continue to serve as a natural storehouse of goods and services into the future. They help in the conservation of indigenous species that are resistant to pests, diseases and pathogens, environmental stresses and nutrient loss.

Biological diversity is critical for the maintenance of ecosystem. Each species in the forest plays a fundamental role in the maintenance of the forest ecosystem. Tropical forests are one of the main repositories of global biodiversity (Rennolls \& Reynold, 2007; Sarkar \& Devi, 2017). Unfortunately, this biodiversity is under threat as a result of forest degradation and deforestation. The likely impact of degradation and deforestation in tropical rainforest has direct environmental and biological implications (Barua et al., 2018).

It is important to quantify the tree species diversity of protected areas so as to provide useful information on the genetic resources available, estimate the level of adaptation to the environment, their ecological significance and the efficacy of these protected areas in biodiversity conservation. For the protection of forests from degradation and deforestation, it is vital to investigate the present status of species diversity as it will provide direction for the management of forest areas (Bajpai et al., 2012; Nurudeen et al., 2017; Bajpai et al., 2018). Information from this quantitative inventory will provide a valuable reference for forest assessment and improve our knowledge in identification of ecologically useful species as well as species of special concern. Therefore, this study was carried out to assess the present status of protected areas in Akure Forest Reserve and their efficacy as biodiversity hotspot. This was achieved through field inventory and on-the-spot tree diversity assessment. 


\section{METHODOLOGY}

\section{The study area}

The three protected areas i.e. Strict Nature Reserve (SNR), Buffer zone (BZ) and the Enrichment Planting (EP) are located in Akure forest Reserve. The reserve is geographically located in a humid rainforest zone of Akure South Local government area of Ondo State, Nigeria. It lies between latitudes $7^{\circ} 16^{\prime}$ and $7^{\circ} 18^{\prime} \mathrm{N}$ of the Equator and longitudes $5^{\circ} 9^{\prime}$ and $5^{\circ} 11^{\prime} \mathrm{E}$ of the Greenwich Meridian. It was constituted as a reserve in 1936 and the total land area covered is 69.93 $\mathrm{km}^{2}$. Politically, it lies in Ondo State in Southwestern Nigeria. The relief pattern is low lying, elevation ranges from 216 $\mathrm{m}$ to $504 \mathrm{~m}$ and gently undulating in southern part while the northern part is hilly rock outcrops occurring at close intervals. The underlying rock is crystalline and gneiss. It is slightly neutral; $\mathrm{pH}$ of 6.7-7.3 and sandy-loam in nature. The dry season lasts from November to March while the wet season commences from April and ends in October with the highest rainfall records between July and August, Average daily temperature ranges between $21^{\circ} \mathrm{C}$ and $29^{\circ} \mathrm{C}$ almost throughout the year. The mean annual rainfall varies from $2000 \mathrm{~mm}$ in southern area to $1500 \mathrm{~mm}$ in northern area with relative humidity of $80-85 \%$ annually experienced in south-west.

\section{Data collection}

Sample plots demarcation

Systematic sampling design (systematic line transects) was used in plot layout. Two equal plots of size $50 \mathrm{~m} \times 50 \mathrm{~m}$ were alternatively laid on the transect. All woody plants within the transect were enumerated.

\section{Tree enumeration}

All Woody plants with DBH greater than $10 \mathrm{~cm}$ were enumerated. Tree growth variables such as the diameter at the base $\left(D_{b}\right)$, Diameter at breast height $(\mathrm{DBH})$, diameter at the middle $\left(\mathrm{D}_{\mathrm{m}}\right)$, Diameter at the top $\left(\mathrm{D}_{t}\right)$ and height were measured for basal area and volume estimation.

\section{Biodiversity indices and tree species classification}

The botanical name of every living trees encountered in each sample plot was recorded. Where a tree's botanical name is not known immediately, such a tree was identified by its commercial or local name. Such commercial or local names were translated to correct botanical names using Gbile (1984) and Keay (1989). Frequencies of occurrence were obtained for species abundance or richness. This was repeated for all the trees encountered in the sample plots. All trees were assigned to families and the number of species in each family were obtained.

\section{Method of data analysis}

Basal area estimation

Basal area per hectare was obtained by multiplying the mean plot basal area by the number of plots per hectare

$$
\mathrm{BA}=\frac{\pi \mathrm{D}^{2}}{4}
$$

Where, BA= Basal area $\left(\mathrm{m}^{2}\right) ; \mathrm{D}=$ Diameter at breast height $(\mathrm{cm}) ; \pi=$ Pie (3.142).

\section{Volume estimation}

The volume of individual trees was estimated using the Newton formula (Husch et. al., 2003).

$$
\mathrm{V}=\frac{\pi \mathrm{h}}{24}\left(\mathrm{D}_{\mathrm{b}}^{2}+4 \mathrm{D}_{\mathrm{m}}^{2}+\mathrm{D}_{\mathrm{t}}^{2}\right)
$$

Where, $V=$ Volume of tree $\left(\mathrm{m}^{3}\right) ; \mathrm{D}_{\mathrm{b}}=$ Diameter at the base $(\mathrm{m}) ; \mathrm{D}_{\mathrm{m}}=$ Diameter at the middle $(\mathrm{m}) ; \mathrm{D}_{\mathrm{t}}=$ Diameter at the top (m); $\mathrm{H}=$ height $(\mathrm{m})$.

Volume per hectare was obtained by multiplying the number of plots per hectare.

\section{Biodiversity indices}

\section{Important value index}

Important value index was computed as,

$$
\mathrm{IVI}=\frac{(\mathrm{RD}+\mathrm{RDo})}{2}
$$

Where, $\mathrm{RD}=$ Relative density of the Species; $\mathrm{RD}_{\mathrm{o}}=$ Relative dominance of Species.

Species relative density $(R D)$

Species relative density, which is an index for assessing species relative distribution, was computed as,

$$
\mathrm{RD}=\frac{\mathrm{n}_{\mathrm{i}}}{N} \times 100
$$

Where, $\mathrm{RD}=$ Relative density of the species; $\mathrm{n}_{i}=$ Number of individuals per species; $N=$ Total number of all 
individual trees of all species in the entire population.

\section{Species relative dominance}

Species relative dominance was estimated as,

$$
\mathrm{RDo}=\frac{\sum \mathrm{Ba}_{\mathrm{i}}}{\sum \mathrm{Ba}_{\mathrm{n}}} \times 100
$$

Where, $\mathrm{Ba}_{\mathrm{i}}=$ basal area of individual tree belonging to the $\mathrm{i}^{\text {th }}$ species; $\mathrm{Ba}_{\mathrm{n}}=$ stand basal area.

Shannon-Wiener Diversity Index

Species diversity index was calculated using the Shannon-Wiener diversity index,

$$
\mathrm{H}^{\prime}=-\sum_{i=1}^{\mathrm{S}} P_{i} \operatorname{In}\left(P_{i}\right)
$$

Where, $H^{\prime}=$ Shannon diversity index; $\mathrm{S}=$ Total number of species in the community; $\mathrm{P}_{\mathrm{i}}=$ Proportion $\mathrm{S}$ (species in the family) made up of the ith species; $\ln =$ Natural logarithm.

The Species evenness ( $E$ )

Species evenness in each plot was determined using Shannon's equitability $\left(\mathrm{E}_{\mathrm{H}}\right)$,

$$
\mathrm{E}_{\mathrm{H}}=\frac{\mathrm{H}^{\prime}}{\mathrm{H}_{\mathrm{Max}}}=\frac{\sum_{i=1}^{S} P_{i} \operatorname{In}\left(P_{i}\right)}{\operatorname{In}(\mathrm{S})}
$$

\section{RESULTS}

The results of the study revealed that Funtumia elastica (Preuss) Stapf was the tree species with highest frequency of occurrence in the SNR with 48 stems. So, it could be regarded as the dominant species in the site. This was followed by Sterculia rhinopetala K. Schum with 32 stems. Annonidium manii (Oliv.) Engl. \& Diels was the most abundant tree species in buffer zone and Enrichment Planting represented by 40 and 12 stems respectively. Some of the species represented by four stems in the buffer zone are Alstonia boonei De Wild, Brachystegia eurycoma Harms, Celtis mildbreadii Engl., Chrysophyllum purpuchrum Mildbr. ex Hutch. \& Dalziel, Cola hispida Brenan \& Keay, Khaya grandifoliola C. DC., Milicia excelsa (Welw.) C. Berg, Margaritaria discoidea (Baill.) G.L. Webster and Ricinodendron heudelotii (Baill.) Heckel. In enrichment planting site, Strombosia postulata Oliv. and Trilepisium madagascariense DC. are represented by 6 and 8 stems respectively. Some of the tress species with just 2 stems ha ${ }^{-1}$ include; Baphia nitida Lodd., Boscia senegalensis (Pers.) Lam. ex Poir., Triplochyton scleroxylon K. Schum, Milicia excelsa, Entadrophragma cylindricum C. DC. (Table 1). Table 2 shows the Shannon wiener Index values obtained in the three protected areas. In Strict Nature Reserve, Funtumia elastica had the highest Shannon Wiener value of 0.31 , followed by Sterculia rhinopetala with an index of 0.26. Some of the tree species with Shannon Wiener value of 0.02 are Alstonia boonei, Brachystegia eurycoma, Celtis philippensis Planch, Daniellia ogea (Harms) Holland, Diospyros mespiliformis Hochst. ex A.DC. and Diospyros dendo Welw., Annonidium manii had the highest Shannon Wiener value of 0.32 in the buffer zone followed by Cola gigantea A. Chev. with a value of 0.22. Some of the species with Shannon Wiener value of 0.05 include; Cola acuminata (P. Beauv.) Schott \& Endl., Khaya grandifoliola, Trichilia welwitschii C. DC., Garcinia kola Heckel, Gmelina arborea Roxb., Greenwayodendron sp., Lecaniodiscus cupanioides Planch. ex Benth. Enrichment planting site was dominated by the species of Annonidium manii with a Shannon Wiener value of 0.3. Shannon Wiener values of 0.21 and 0.24 were obtained for Strombosia pustulata Oliv. and Trilepisium madagascariense.

The summary of tree growth variables \& biodiversity indices is presented in table 3 . The number of stems per hectare follows the order of SNR: 264 > Buffer Zone: 198 > Enrichment Planting: 72. A high volume of the trees per hectare was recorded in the SNR $\left(461.74 \mathrm{~m}^{3}\right)$, followed by Buffer zone $\left(424.46 \mathrm{~m}^{3}\right)$ and Enrichment Planting (138.28 $\mathrm{m}^{3}$ ). The basal area per hectare obtained in this study ranges from $14.35 \mathrm{~m}^{2}$ for Enrichment Planting site to $60.62 \mathrm{~m}^{2}$ recorded for SNR. The Shannon-Wiener Diversity values of 3.10, 2.97, and 3.0 were recorded for SNR, Buffer zone and Enrichment Planting respectively. The Buffer Zone and SNR both had evenness of 0.86 and 0.93 was obtained for Enrichment Planting site. In SNR, the highest IVI was recorded for Funtumia elastica (14.37) while the lowest IVI was recorded for Milicia excelsa and Diospyros dendo with an IVI of 0.43 each. Annonidium manii had the highest IVI in the buffer zone with IVI of 22.85, this was followed by Celtis zenkeri Engl. and Cola gigantea with an IVI of 10.83 and 10.24 respectively. Species with high IVI in the Enrichment planting site are Annonidium manii with IVI of 20.8, Trilepisium madagascariense with IVI of 13.71 and Cola acuminata with IVI of 4.40. Only Brachystegia nigerica Hoyle \& A.P.D. Jones had IVI that is less than 2 (Table 4). Analysis of Variance (ANOVA) for biodiversity indices obtained in the study sites is presented in table 5. The results revealed a significant difference $(\mathrm{P}<0.05)$ in the Species 
Table 1. Tree species distribution in the three protected areas i.e. Strict Nature Reserve (SNR), Buffer zone (BZ) and the Enrichment Planting (EP).

\begin{tabular}{|c|c|c|c|c|c|}
\hline \multirow[t]{2}{*}{ S.N. } & \multirow[t]{2}{*}{ Species } & \multirow[t]{2}{*}{ Family } & \multicolumn{3}{|c|}{ Number of stems ha ${ }^{-1}$} \\
\hline & & & SNR & $\mathbf{B Z}$ & EP \\
\hline 1 & Alstonia boonei De Wild & Apocynaceae & 2 & 4 & 0 \\
\hline 2 & Annonidium manii (Oliv.) Engl. \& Diels & Annonaceae & 10 & 40 & 12 \\
\hline 3 & Baphia nitida Lodd. & Fabaceae & 0 & 0 & 2 \\
\hline 4 & Boscia senegalensis (Pers.) Lam. ex Poir. & Moraceae & 0 & 0 & 2 \\
\hline 5 & Brachystegia eurycoma Harms & Leguminosae & 2 & 4 & 0 \\
\hline 6 & Brachystegia nigerica Hoyle \& A.P.D. Jones & Leguminosae & 0 & 0 & 2 \\
\hline 7 & Ceiba pentandra (L.) Gaertn. & Malvaceae & 0 & 0 & 2 \\
\hline 8 & Celtis mildbreadii Engl. & Ulmaceae & 8 & 4 & 0 \\
\hline 9 & Celtis philippensis Planch & Cannabaceae & 2 & 0 & 2 \\
\hline 10 & Celtis sp. & Ulmaceae & 0 & 0 & 2 \\
\hline 11 & Celtis zenkeri Engl. & Ulmaceae & 14 & 16 & 0 \\
\hline 12 & Chrysophyllum albidum G. Don & Sapotaceae & 0 & 0 & 2 \\
\hline 13 & Chrysophyllum purpuchrum Mildbr. ex Hutch. \& Dalziel & Sapotaceae & 4 & 4 & 0 \\
\hline 14 & Cola acuminata (P. Beauv.) Schott \& Endl. & Malvaceae & 2 & 2 & 0 \\
\hline 15 & Cola gigantea A. Chev & Sterculiaceae & 10 & 18 & 4 \\
\hline 16 & Cola hispida Brenan \& Keay & Sterculiaceae & 0 & 4 & 2 \\
\hline 17 & Cola nitida Schott \& Endl. & Malvaceae & 8 & 0 & 0 \\
\hline 18 & Cordia millenii Baker & Boraginaceae & 4 & 0 & 0 \\
\hline 19 & Daniellia ogea (Harms) Holland & Leguminosae & 2 & 0 & 0 \\
\hline 20 & Diospyros dendo Welw. & Ebeneceae & 0 & 10 & 0 \\
\hline 21 & Diospyros mespiliformis Hochst. ex A.DC. & Ebeneceae & 2 & 0 & 0 \\
\hline 22 & Diesplacia suberulcapa Juan Rulfo & Ebeneceae & 0 & 2 & 0 \\
\hline 23 & Diospyros borneensis Hiern & Ebeneceae & 0 & 0 & 2 \\
\hline 24 & Entandrophragma angolense (Welw.) C. DC. & Meliaceae & 0 & 12 & 0 \\
\hline 25 & Entadrophragma cylindricum C. DC. & Meliaceae & 0 & 0 & 2 \\
\hline 26 & Entandrophragma utile (Dawe \& Sprague) Sprague & Meliaceae & 8 & 0 & 0 \\
\hline 27 & Funtumia elastica (Preuss) Stapf & Apocynaceae & 48 & 2 & 2 \\
\hline 28 & Garcinia kola Heckel & Clusiaceae & 0 & 2 & 2 \\
\hline 29 & Gmelina arborea Roxb. & Lamiaceae & 0 & 2 & 0 \\
\hline 30 & Greenwayodendron sp. & Annonaceae & 0 & 2 & 0 \\
\hline 31 & Khaya grandifoliola C. DC. & Meliaceae & 2 & 2 & 0 \\
\hline 32 & Lecaniodiscus cupanioides Planch. ex Benth. & Sapindaceae & 0 & 2 & 0 \\
\hline 33 & Malacantha alnifolia (Baker) Pierre & Sapotaceae & 6 & 0 & 0 \\
\hline 34 & Mallotus oppositifolius (Gieseler) Mull. Arg. & Euphorbiaceae & 0 & 0 & 2 \\
\hline 35 & Mansonia altissima A. Chev & Sterculiaceae & 20 & 10 & 2 \\
\hline 36 & Margaritaria discoidea (Baill.) G.L. Webster & Phyllanthaceae & 0 & 4 & 0 \\
\hline 37 & Milicia excelsa (Welw.) C. Berg & Moraceae & 2 & 0 & 2 \\
\hline 38 & Musanga cecropioides $\mathrm{R} . \mathrm{Br}$. & Urticaceae & 4 & 0 & 0 \\
\hline 39 & Myrianthus arboreus P. Beauv. & Urticaceae & 2 & 2 & 0 \\
\hline 40 & Picralima nitida (Stapf) T.Durand \& H.Durand & Apocynaceae & 2 & 0 & 0 \\
\hline 41 & Pterocarpus osun Craib & Leguminosae & 2 & 6 & 2 \\
\hline 42 & Pterocarpus sp. & Leguminosae & 2 & 2 & 0 \\
\hline 43 & Pterygota macrocarpa K. Schum & Malvaceae & 12 & 0 & 0 \\
\hline 44 & Pycnanthus angolensis (Welw.) Warb. & Myristicaceae & 2 & 2 & 0 \\
\hline 45 & Ricinodendron heudelotii (Baill.) Heckel & Euphorbiaceae & 0 & 4 & 0 \\
\hline 46 & Spathodea campanulata P. Beauv. & Bignoniaceae & 0 & 0 & 2 \\
\hline 47 & Sterculia oblongata $\mathrm{R} . \mathrm{Br}$. & Sterculiaceae & 2 & 0 & 0 \\
\hline 48 & Sterculia rhinopetala $\mathrm{K}$. Schum & Sterculiaceae & 32 & 6 & 2 \\
\hline 49 & Strombosia postulata Oliv & Olacaceae & 6 & 10 & 6 \\
\hline 50 & Terminalia ivorensis A. Chev. & Combretaceae & 2 & 0 & 0 \\
\hline 51 & Terminalia superba Engl. \& Diels & Combretaceae & 10 & 0 & 0 \\
\hline 52 & Trichilia heudelotii Planch. ex Oliv. & Meliaceae & 8 & 0 & 0 \\
\hline 53 & Trichilia sp. & Meliaceae & 0 & 2 & 0 \\
\hline 54 & Trichilia welwitschii C. DC. & Meliaceae & 4 & 2 & 2 \\
\hline 55 & Trilepisium madagascariense DC. & Moraceae & 6 & 14 & 8 \\
\hline 56 & Triplochyton scleroxylon $\mathrm{K}$. Schum & Malvaceae & 8 & 6 & 2 \\
\hline 57 & Zanthoxylum utile Huang. & Rutaceae & 2 & 0 & 0 \\
\hline \multirow[t]{2}{*}{58} & Zanthoxylum zanthoxyloides (Lam.) Zepern. \& Timler & Rutaceae & 2 & 0 & 0 \\
\hline & & Total & 264 & 198 & 72 \\
\hline
\end{tabular}


Table 2. Shannon wiener index for the three protected areas i.e. Strict Nature Reserve (SNR), Buffer zone (BZ) and the Enrichment Planting (EP).

\begin{tabular}{|c|c|c|c|c|c|c|}
\hline \multirow[t]{2}{*}{ Tree species } & \multicolumn{2}{|c|}{ BZ } & \multicolumn{2}{|c|}{ SNR } & \multicolumn{2}{|l|}{ EP } \\
\hline & Freq. ha $^{-1}$ & $\mathbf{H}^{1}$ & Freq. ha $^{-1}$ & $\mathbf{H}^{1}$ & Freq. ha $^{-1}$ & $\mathbf{H}^{1}$ \\
\hline Alstonia boonei De Wild & 4 & -0.08 & 2 & -0.04 & - & - \\
\hline Annonidium manii (Oliv.) Engl. \& Diels & 40 & -0.32 & 10 & -0.12 & 12 & -0.3 \\
\hline Baphia nitida Lodd. & - & - & - & - & 2 & -0.1 \\
\hline Boscia senegalensis (Pers.) Lam. ex Poir. & - & - & - & - & 2 & -0.1 \\
\hline Brachystegia nigerica Hoyle \& A.P.D. Jones & - & - & - & - & 2 & -0.1 \\
\hline Brachystegia eurycoma Harms & 4 & -0.08 & 2 & -0.04 & - & - \\
\hline Ceiba pentandra (L.) Gaertn. & - & - & - & - & 2 & -0.1 \\
\hline Celtis mildbreadii Engl. & 4 & -0.08 & 8 & -0.11 & - & - \\
\hline Celtis philippensis Planch & - & - & 2 & -0.04 & 2 & -0.1 \\
\hline Celtis zenkeri Engl. & 16 & -0.2 & 14 & -0.16 & 2 & -0.1 \\
\hline Chrysophyllum albidum G. Don & - & - & - & - & 2 & -0.1 \\
\hline Chrysophyllum purpuchrum Mildbr. ex Hutch \& Dalziel & 4 & -0.08 & 4 & -0.06 & - & - \\
\hline Cola acuminata (P. Beauv.) Schott \& Endl. & 2 & -0.05 & 2 & -0.04 & - & - \\
\hline Cola gigantea A. Chev. & 18 & -0.22 & 10 & -0.12 & 4 & -0.16 \\
\hline Cola hispida Brenan \& Keay. & 4 & -0.08 & - & - & 2 & -0.1 \\
\hline Diospyros borneensis Hiern & - & - & - & - & 2 & -0.1 \\
\hline Cola nitida Schott \& Endl. & - & - & 8 & -0.11 & - & - \\
\hline Cordia millenii Baker & - & - & 4 & -0.06 & - & - \\
\hline Daniellia ogea (Harms) Holland & - & - & 2 & -0.04 & - & - \\
\hline Diospyros mespiliformis Hochst. ex A.DC. & - & - & 2 & -0.04 & - & - \\
\hline Diospyros dendo Welw. & 8 & -0.13 & 2 & -0.04 & - & - \\
\hline Diesplacia suberulcapa Juan Rulfo & 2 & -0.05 & - & - & - & - \\
\hline Entadrophragma cylindricum C. DC. & - & - & - & - & 2 & -0.1 \\
\hline Entandrophragma utile (Dawe \& Sprague) Sprague & - & - & 8 & -0.11 & - & - \\
\hline Entandrophragma angolense (Welw.) C. DC. & 12 & -0.17 & - & - & - & - \\
\hline Funtumia elastica (Preuss) Stapf & 2 & -0.05 & 48 & -0.31 & 2 & -0.1 \\
\hline Garcinia kola Heckel & 2 & -0.05 & - & - & 2 & -0.1 \\
\hline Gmelina arborea Roxb. & 2 & -0.05 & - & - & - & - \\
\hline Greenwayodendron sp. & 2 & -0.05 & - & - & - & - \\
\hline Khaya grandifoliola C. DC. & 2 & -0.05 & 2 & -0.04 & - & - \\
\hline Lecaniodiscus cupanioides Planch. ex Benth. & 2 & -0.05 & - & - & - & - \\
\hline Malacantha alnifolia (Baker) Pierre & - & - & 6 & -0.09 & - & - \\
\hline Mallotus oppositifolius (Gieseler) Mull. Arg. & - & - & - & - & 2 & -0.1 \\
\hline Mansonia altissima A. Chev & 10 & -0.15 & 20 & -0.2 & 2 & -0.1 \\
\hline Margaritaria discoidea (Baill.) G.L. Webster & 2 & -0.05 & - & - & - & - \\
\hline Milicia excelsa (Welw.) C. Berg & - & - & 2 & -0.04 & 2 & -0.1 \\
\hline Musanga cecropioides $\mathrm{R} . \mathrm{Br}$. & - & - & 4 & -0.06 & - & - \\
\hline Myrianthus arboreus $\mathrm{P}$. Beauv & 2 & -0.05 & - & - & 2 & -0.1 \\
\hline Picralima nitida (Stapf) T.Durand \& H.Durand & - & - & 2 & -0.04 & - & - \\
\hline Pterocarpus osun Craib & 6 & -0.11 & 2 & -0.04 & 2 & -0.1 \\
\hline Pterocarpus sp. & 2 & -0.05 & 2 & -0.04 & - & - \\
\hline Pterygota macrocarpa K. Schum & - & - & 12 & -0.14 & - & - \\
\hline Pycnanthus angolensis (Welw.) Warb. & 2 & -0.05 & 2 & -0.04 & - & - \\
\hline Ricinodendron heudelotii (Baill.) Heckel & 4 & -0.08 & - & - & - & - \\
\hline Spathodea campanulata P. Beauv. & - & - & - & - & 2 & -0.1 \\
\hline Sterculia oblongata $\mathrm{R} . \mathrm{Br}$. & - & - & 2 & -0.04 & & - \\
\hline Sterculia rhinopetala $\mathrm{K}$. Schum & 6 & -0.11 & 32 & -0.26 & 2 & -0.1 \\
\hline Strombosia postulata Oliv. & 10 & -0.15 & 6 & -0.09 & 6 & -0.21 \\
\hline Trichilia sp. & 2 & -0.05 & - & - & - & - \\
\hline Terminalia ivorensis A. Chev. & - & - & 2 & -0.04 & - & - \\
\hline Terminalia superba Engl. \& Diels & - & - & 10 & -0.12 & - & - \\
\hline Trichilia heudelotii Planch. ex Oliv. & - & - & 8 & -0.11 & - & - \\
\hline Trichilia welwitschii C. DC. & 2 & -0.05 & 4 & -0.06 & 2 & -0.1 \\
\hline Trilepisium madagascariense DC. & 14 & -0.19 & 6 & -0.09 & 8 & -0.24 \\
\hline Triplochyton scleroxylon $\mathrm{K}$. Schum & 6 & -0.11 & 8 & -0.11 & 2 & -0.1 \\
\hline Zanthoxylum utile Huang & - & - & 2 & -0.04 & - & - \\
\hline Zanthoxylum zanthoxyloides (Lam.) Zepern. \& Timler & - & - & 2 & -0.04 & - & - \\
\hline Total & 198 & -2.97 & 264 & -3.1 & 72 & -3 \\
\hline
\end{tabular}


Table 3. Summary of tree growth variables \& biodiversity indices from Strict Nature Reserve (SNR), Buffer zone (BZ) and the Enrichment Planting (EP).

\begin{tabular}{|c|c|c|c|}
\hline Growth Variables \& Biodiversity Indices & SNR & $\mathbf{B Z}$ & EP \\
\hline No of trees ha ${ }^{-1}$ & 264 & 198 & 72 \\
\hline No of species & 37 & 31 & 25 \\
\hline Volume per hectare $\left(\mathrm{m}^{3}\right)$ & 461.74 & 424.46 & 138.28 \\
\hline Basal area per hectare $\left(\mathrm{m}^{2}\right)$ & 60.62 & 36.48 & 14.35 \\
\hline Mean DBH $(\mathrm{cm})$ & 30.0 & 31.0 & 24.7 \\
\hline Mean height (m) & 15.70 & 14.38 & 13.00 \\
\hline Shannon Wiener index $\left(\mathrm{H}^{1}\right)$ & 3.10 & 2.97 & 3.00 \\
\hline Pieolus Species Evenness & 0.86 & 0.86 & 0.93 \\
\hline
\end{tabular}

Table 4. Importance value index (IVI) of tree species in the study sites i.e. Strict Nature Reserve (SNR), Buffer zone (BZ) and the Enrichment Planting (EP).

\begin{tabular}{|c|c|c|c|c|c|c|c|c|c|}
\hline \multirow[t]{2}{*}{ Tree species } & \multicolumn{3}{|c|}{$\mathbf{B Z}$} & \multicolumn{3}{|c|}{ SNR } & \multicolumn{3}{|c|}{$\mathbf{E P}$} \\
\hline & RD\% & RDO\% & IVI & RD\% & RDO\% & IVI & RD\% & RDO\% & $\overline{\text { IVI }}$ \\
\hline Alstonia boonei De Wild & 2.02 & 1.38 & 1.7 & 0.76 & 0.16 & 0.46 & - & - & \\
\hline Anonidium mannii (Oliv.) Engl. \& Diels & 20.2 & 25.49 & 22.85 & 3.79 & 1.41 & 2.60 & 16.67 & 24.91 & 20.8 \\
\hline Baphia nitida Lodd. & - & - & - & - & - & - & 2.78 & 2.35 & 2.57 \\
\hline Boscia senegalensis (Pers.) Lam. ex Poir. & - & - & - & - & - & - & 2.78 & 1.76 & 2.27 \\
\hline Brachystegia nigerica Hoyle \& A.P.D. Jones & - & - & - & - & - & - & 2.78 & 1.03 & 1.91 \\
\hline Brachystegia eurycoma Harms & 2.02 & 1.37 & 1.70 & 0.76 & 0.16 & 0.46 & - & - & \\
\hline Ceiba pentandra (L.) Gaertn. & - & - & - & - & - & - & 2.78 & 2.06 & 2.42 \\
\hline Celtis mildbreadii Engl. & 2.02 & 0.85 & 1.435 & 3.03 & 2.83 & 2.93 & - & - & . \\
\hline Celtis philippensis Planch & - & - & - & 0.76 & 0.5 & 0.63 & 2.78 & 1.22 & 2 \\
\hline Celtis sp. & - & - & - & - & - & - & 2.78 & 5.39 & 4.09 \\
\hline Celtis zenkeri Engl. & 8.08 & 13.58 & 10.83 & 5.3 & 1.45 & 3.38 & - & - & \\
\hline Chrysophyllum albidum G. Don & - & - & - & - & - & - & 2.78 & 1.35 & 2.07 \\
\hline Chrysophyllum purpuchrum Mildbr. ex Hutch. \& Dalziel & 2.02 & 2.29 & 2.16 & 1.52 & 0.94 & 1.23 & - & - & - \\
\hline Cola acuminata (P. Beauv.) Schott \& Endl. & 1.01 & 0.34 & 0.68 & 0.76 & 0.17 & 0.47 & - & - & - \\
\hline Cola gigantea A. Chev & 9.09 & 11.39 & 10.24 & 3.79 & 4.06 & 3.93 & 5.56 & 3.23 & 4.40 \\
\hline Cola hispida Brenan \& Keay & 2.02 & 0.47 & 1.25 & & & & 2.78 & 1.62 & 2.2 \\
\hline Cola nitida Schott \& Endl. & - & - & - & 3.03 & 0.7 & 1.87 & - & - & - \\
\hline Cordia millenii Baker & - & - & - & 1.52 & 7.76 & 4.64 & - & - & - \\
\hline Daniellia ogea (Harms) Holland & - & - & - & 0.76 & 1.24 & 1.00 & - & - & - \\
\hline Diospyros mespiliformis Hochst. ex A.DC. & - & - & - & 0.76 & 0.22 & 0.49 & - & - & - \\
\hline Diesplacia suberulcapa Juan Rulfo & 1.01 & 0.34 & 0.675 & - & - & - & - & - & - \\
\hline Diospyros borneensis Hiern & - & - & - & - & - & - & 2.78 & 2.21 & 2.495 \\
\hline Diospyros dendo Welw. & 4.04 & 2 & 3.02 & 0.76 & 0.1 & 0.43 & - & - & - \\
\hline Entandrophragma angolense (Welw.) C. DC. & 6.06 & 2.91 & 4.485 & - & - & - & - & - & 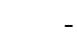 \\
\hline Entadrophragma cylindricum C. DC. & - & - & - & - & - & - & 2.78 & 1.39 & 2.085 \\
\hline Entandrophragma utile (Dawe \& Sprague) Sprague & - & - & - & 3.04 & 6.18 & 4.61 & - & - & - \\
\hline Funtumia elastica (Preuss) Stapf & 1.01 & 0.18 & 0.595 & 18.18 & 10.55 & 14.37 & 2.78 & 1.86 & 2.32 \\
\hline Garcinia kola Heckel & 1.01 & 0.19 & 0.6 & - & - & - & 2.78 & 3.67 & 3.225 \\
\hline Gmelina arborea Roxb. & 1.01 & 1.34 & 1.175 & - & - & - & - & - & - \\
\hline Greenwayodendron sp. & 1.01 & 0.69 & 0.85 & - & - & - & - & - & \\
\hline Khaya grandifoliola C. DC. & 1.01 & 1.05 & 1.03 & 0.76 & 0.18 & 0.47 & - & - & - \\
\hline Lecaniodiscus cupanioides Planch. ex Benth. & 1.01 & 0.91 & 0.96 & - & - & & - & - & - \\
\hline Malacantha alnifolia (Baker) Pierre & - & - & - & 2.27 & 0.32 & 1.30 & - & - & - \\
\hline Mallotus oppositifolius (Gieseler) Mull. Arg. & - & - & - & - & - & - & 2.78 & 2 & 2.39 \\
\hline Mansonia altissima A. Chev & 5.05 & 8.47 & 6.76 & 7.58 & 5.86 & 6.72 & 2.78 & 2.47 & 2.625 \\
\hline Margaritaria discoidea (Baill.) G.L. Webster & 1.01 & 0.35 & 0.68 & - & - & - & - & - & \\
\hline Milicia excelsa (Welw.) C. Berg & - & - & - & 0.76 & 0.09 & 0.43 & 2.78 & 3.49 & 3.135 \\
\hline Musanga cecropioides $\mathrm{R} . \mathrm{Br}$. & - & - & - & 1.52 & 0.95 & 1.24 & & & 0 \\
\hline Myrianthus arboreus $\mathrm{P}$. Beauv & 1.01 & 1.21 & 1.11 & & & & 2.78 & 1.33 & 2.055 \\
\hline Picralima nitida (Stapf) T. Durand \& H. Durand & - & - & - & 0.76 & 0.2 & 0.48 & - & - & \\
\hline Pterocarpus osun Craib & 3.03 & 1.46 & 2.245 & 0.76 & 0.09 & 0.43 & 2.78 & 2.53 & 2.655 \\
\hline Pterocarpus sp. & 1.01 & 0.14 & 0.575 & 0.76 & 0.2 & 0.48 & - & - & \\
\hline Pterygota macrocarpa K. Schum & & & & 4.55 & 12.65 & 8.60 & - & - & - \\
\hline Pycnanthus angolensis (Welw.) Warb. & 1.01 & 1.88 & 1.445 & 0.76 & 0.21 & 0.49 & - & - & - \\
\hline Ricinodendron heudelotii (Baill.) Heckel & 2.02 & 0.38 & 1.2 & - & - & - & - & - & - \\
\hline Spathodea campanulata P. Beauv. & - & - & - & - & - & - & 2.78 & 5.32 & 4.05 \\
\hline Sterculia oblongata $\mathrm{R} . \mathrm{Br}$. & - & - & - & 0.76 & 1.28 & 1.02 & - & - & . \\
\hline
\end{tabular}




\begin{tabular}{|c|c|c|c|c|c|c|c|c|c|}
\hline Sterculia rhinopetala K. Schum & 3.03 & 2.54 & 2.785 & 12.12 & 13.17 & 12.65 & 2.78 & 2.15 & 2.465 \\
\hline Strombosia pustulata Oliv. & 5.05 & 5.32 & 5.185 & 2.27 & 0.79 & 1.53 & 8.33 & 5.54 & 6.935 \\
\hline Terminalia ivorensis A. Chev. & - & - & - & 0.76 & 2.18 & 1.47 & - & - & . \\
\hline Terminalia superba Engl. \& Diels & - & - & - & 3.79 & 8.69 & 6.24 & - & - & - \\
\hline Trichilia heudelotii Planch. ex Oliv. & - & - & - & 3.03 & 0.67 & 1.85 & - & - & - \\
\hline Trichilia sp. & 1.01 & 0.13 & 0.57 & - & - & - & - & - & - \\
\hline Trichilia welwitschii C. DC. & 1.01 & 0.19 & 0.6 & 1.52 & 0.16 & 0.84 & 2.78 & 2.21 & 2.495 \\
\hline Trilepisium madagascariense DC. & 7.07 & 8.17 & 7.62 & 2.27 & 1.02 & 1.65 & 11.11 & 16.31 & 13.71 \\
\hline Triplochyton scleroxylon K. Schum & 3.03 & 3.01 & 3.02 & 3.03 & 12.01 & 7.52 & 2.78 & 2.53 & 2.655 \\
\hline Zanthoxylum utile Huang & - & - & - & 0.76 & 0.23 & 0.50 & - & - & - \\
\hline Zanthoxylum zanthoxyloides (Lam.) Zepern. \& Timler & - & - & - & 0.76 & 0.66 & 0.71 & - & - & - \\
\hline
\end{tabular}

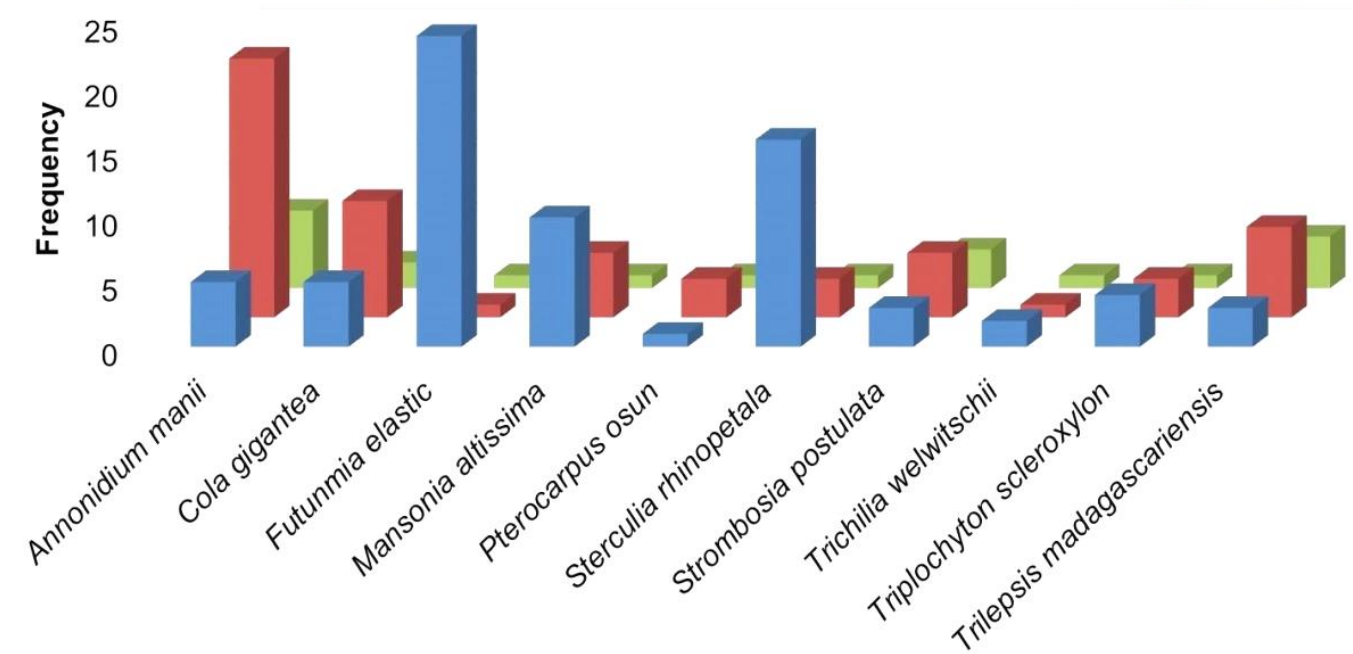

Figure 1. Species frequency in the study sites i.e. Strict Nature Reserve (SNR), Buffer zone (BZ) and the Enrichment Planting (EP).

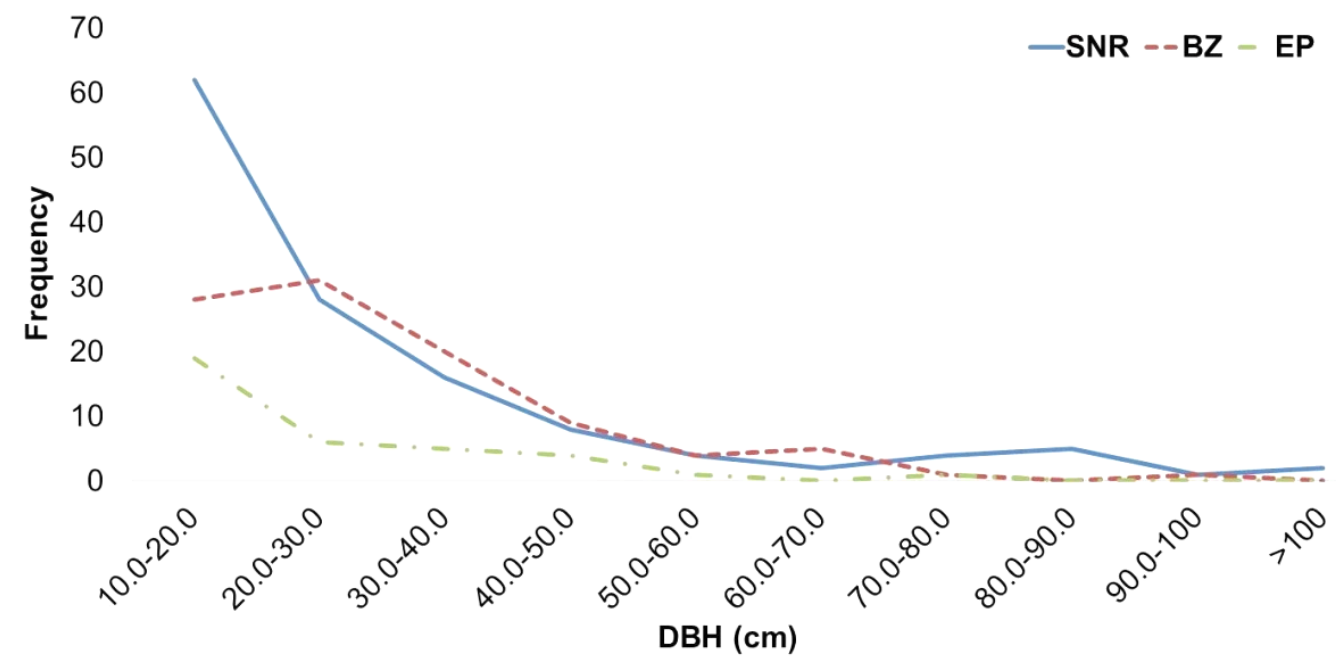

Figure 2. Stems distribution based on DBH class in the study sites i.e. Strict Nature Reserve (SNR), Buffer zone (BZ) and the Enrichment Planting (EP).

evenness and Shannon Wiener index of the three protected areas but no significant difference was observed in their Simpson Concentration. Ten tree species were common to the three protected areas, Annonidium manii, Cola gigantea, Funtumia elastica, Mansonia altissima A. Chev, Pterocarpus osun Craib, Sterculia rhinopetala, Strombosia postulata, Trichilia welwitschii, Triplochyton scleroxylon and Trilepisium madagascariense. Frequency of these common species in all the three protected areas is presented in figure 1. Stems distribution based on DBH class in the three protected areas is presented in figure 2. Majority of the trees in the SNR fell in the lowest diameter class. Twenty-eight (28) trees fell in the DBH class of 20-30 cm. Only two trees fell in the DBH class above $100 \mathrm{~cm}$. Buffer zone is dominated by trees with diameter class $20-30 \mathrm{~cm}$, followed by trees in the lowest diameter class $(10-20 \mathrm{~cm})$. Only one tree fell in the diameter class of 70-80 cm and 90-100 cm. Most of the trees (18) in Enrichment planting site fell in the lower diameter of $10-20 \mathrm{~cm}$, followed by trees in the diameter class of $20-30 \mathrm{~cm}$. The distribution of trees into height classes is 
presented in figure 3. The highest percentage of trees (61) in the SNR and buffer zone fell in the height class of 10-20m. Only one tree fell in the height class of 30-40 m in Buffer zone and Enrichment Planting site (Fig. 3).

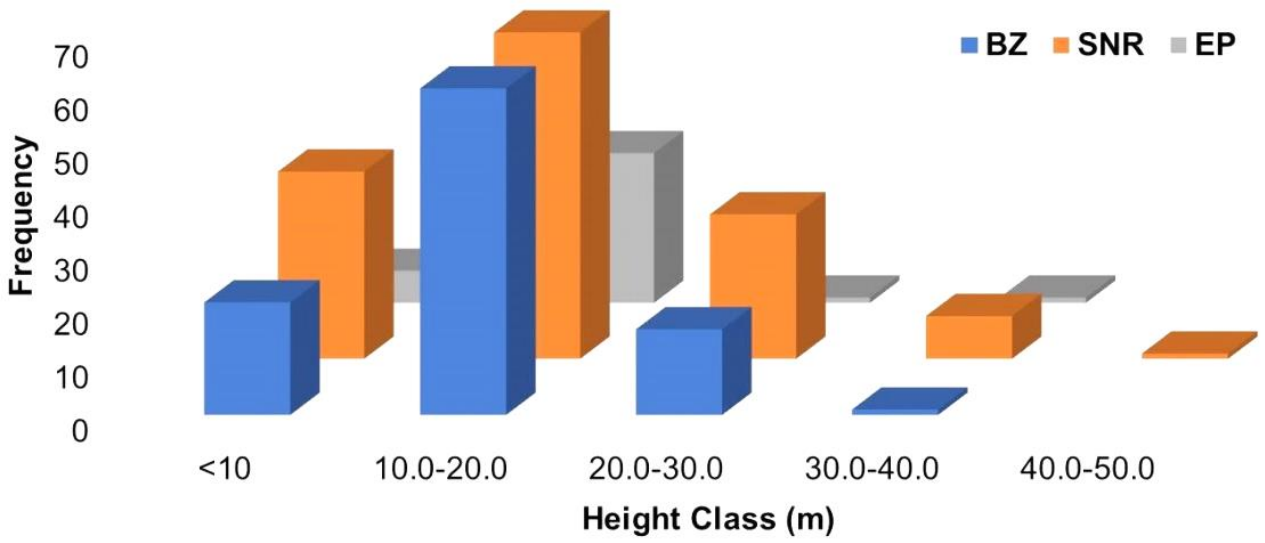

Figure 3. Tree height distribution based on DBH class in the study sites i.e. Strict Nature Reserve (SNR), Buffer zone (BZ) and the Enrichment Planting (EP).

\begin{tabular}{llrrrrr}
\multicolumn{1}{l}{ Table 5. Analysis of Variance (ANOVA) for Biodiversity indices of the study sites. } \\
\hline Biodiversity indices & Source & SS & Df & MS & F & Sig. \\
\hline Species Evenness & Indices & 0.004 & 2 & 0.002 & 6.281 & $0.003^{*}$ \\
& Error & 0.029 & 90 & 0.00 & & \\
Simpson Conc. & Total & $\mathbf{0 . 0 3 3}$ & $\mathbf{9 2}$ & $\mathbf{0 . 0 0}$ & & \\
& Indices & $8.43 E-06$ & 2 & 0.00 & 0.102 & $0.903^{\text {ns }}$ \\
& Error & 0.004 & 90 & 0.00 & & \\
Shannon Wiener index & Total & $\mathbf{0 . 0 0 4}$ & $\mathbf{9 2}$ & $\mathbf{0 . 0 0}$ & & \\
& Indices & 0.026 & 2 & 0.013 & 3.465 & $0.035^{*}$ \\
& Error & 0.335 & 90 & 0.891 & & \\
& Total & $\mathbf{0 . 3 6 1}$ & $\mathbf{9 2}$ & $\mathbf{0 . 0 0 4}$ & & \\
\hline
\end{tabular}

Note: $*$ Significant; ns= Insignificant.

\section{DISCUSSION}

Forests are rich in biological diversity in terms of species, genetic material and ecological processes of all ecosystems (EU, 2008). Forest ecosystem plays vital roles in water cycles, climate change mitigation and carbon sequestration. Protected area is one of the in-situ methods of conservation that are needed to be protected from human anthropogenic activities (Adekunle et al., 2013). The number of trees obtained per hectare in the three protected areas SNR (264), Buffer Zone (198) and Enrichment Planting (72), similar to what was obtained by (Onyekwelu et al., 2007). Most tropical forest ecosystems are rich in floristic composition,this results in a variety of life forms and preservation of global biodiversity (Shi \& Singh, 2002). Biodiversity indices are generated to compare forests compositions and similarities of different species. The higher the value of an ecological index, the higher the species richness (IIRS, 2002).

The Shannon Wiener index values obtained for the three protected areas (SNR 3.16, Buffer Zone 2.97 and Enrichment 3.1) fell within the range of values reported by Adekunle (2006) and Onyekwelu et al. (2008) who revealed that Shannon-Wiener diversity index for tropical rainforests in South-western Nigeria could range between 3.34-3.66 or 2.82-3.31. Species richness is the number of species in a given area of the forest. This was generated to put the three protected areas used in this study on the scale of comparison (Magurran, 2004). The higher the values obtained, the higher the richness of the forest, the high diversity and abundance of the forest denotes that biodiversity is highly conserved (UNEP-WCMC, 2008). The floristic composition and diversity of the protected areas used in this study can be compared to some selected forest reserves in southwest Nigeria (Adekunle et al., 2004), but below what was reported by Kumar et al. (2006) for tropical forests of Garo Hills, India. The high species evenness recorded in the protected areas (PAs) revealed a forest with an evenly distributed number of tree species and stems.

Diameter distribution is an indication of how well the forest is regenerating (Adekunle, 2013). The diameter distribution of trees is used to represent the population structure of forests (Rao et al., 1990). The results of this study revealed that as tree diameter increases, the number of trees decreases and the basal area increases as the DBH increases. The forest reserve is dominated by trees with small diameter, which is common to tropical rainforests. Similar results have been reported by previous workers in other tropical rainforests of Nigeria (Adekunle et al., 2004; Adekunle \& Olagoke, 2008). The reason for few numbers of trees having DBH greater than $50 \mathrm{~cm}$ could be as a result 
of forest degradation activities which might have removed large trees as well as the fact that some trees with large diameter would have been removed through logging activities that has occurred in the past. The basal area per hectare obtained in the three protected area is greater than what was recorded by Maradana \& Owk (2015). The difference in the basal area obtained in each of the PAs can be attributed to species richness, age of stand and level of anthropogenic activities that have occurred in the past (Maradana \& Owk, 2015).

According to Tonolli (2011), tree stem volume is important in forest management and it requires data from field survey. The values obtained for volume per hectare in the buffer zone and Strict nature reserve are less than the value recorded by Adekunle (2006) for an undisturbed site of Akure forest reserve.

The distribution of individual stems into diameter class follows an inverse $\mathbf{J}$ shape, which denotes development of small diameter logs into big diameter trees, a feature that is common to mature natural tropical forests (Adekunle et al., 2013). The height distributions follows a vertical pattern of the forest, this is an indication that the forest will continue to grow till maturity if biodivesity is strictly conserved. However, the presence of trees with large diameter has been reported to be a sign of matured tropical forest (Zheng et al., 2006).

The high volume ha ${ }^{-1}$ obtained especially in the SNR and buffer zone can be attributed to conservation. The study has also shown that the forest ecosystem can be conserved through in situ method of conservation if proper managerial actions are put in place. The species that are present in all the three PAs are typically from the families of Sterculiaceae, Malvaceae, Meliaceae, Moraceae Apocynaceae, Olacaceae and Annonaceae. This finding corroborates the works of Adekunle (2006) and Adekunle et al. (2010) who found that tropical rainforest of southwest Nigeria is dominated by the families of Sterculiaceae, Meliaceae, Moraceae and Ebenaceae.

\section{CONCLUSION AND RECOMMENDATIONS}

Deforestation and degradation are threats to biodiversity conservation. Protected area is one of the insitu methods of conservation and it plays important roles in biodiversity conservation. The floristic composition of these PAs has revealed the efficacy of protected areas in biodiversity conservation. The high biodiversity indices obtained in this study is an indication that the forest will continue to grow till maturity if biodiversity is conserved. The data obtained in this study will provide baseline information for the management of protected areas. This study recommends that conservative measures should be put in place to protect protected areas from deforestation and that more protected area should be established. Forest that have been degraded should be protected from further degradation,

\section{ACKNOWLEDGMENTS}

We wish to thank Forestry Reserve Institute of Nigeria for permitting us to use the reserve for this research and also, we appreciate Prof. V.A.J Adekunle who supervised the work.

\section{REFERENCES}

Adekunle V.A.J. \& Olagoke A.O. (2008). Diversity and biovolume of tree species in natural ecosystem in bitumen-producing area of Ondo State, Nigeria: A baseline study. Biodiversity and Conservation, 17: 2735-2755.

Adekunle V.A.J. (2006) Conservation of tree species diversity in tropical rainforest ecosystem of southwest Nigeria. Journal of Tropical Forest Science 18(2): 91-101.

Adekunle V.A.J., Akindele S.O. \& Fuwape J.A. (2004). Structure and yield models of tropical lowland rainforest ecosystem of southwest Nigeria. Food, Agriculture and Environment, (2): 395-399.

Adekunle V.A.J., Olagoke A.O. \& Akindele S.O. (2013). Tree species diversity and structure of a Nigerian strict nature reserve. International Society for Tropical Ecology, 54(3): 275-289.

Adekunle V.A.J., Olagoke A.O. \& Ogundare L.F. (2010). Rate of timber production in tropical rainforest ecosystem of Nigeria and its implications on sustainable forest management. Journal of Forestry Research, 21(2): 225-230.

Bajpai O., Dutta V., Chaudhary L.B. \& Pandey J. (2018). Key issues and management strategies for the conservation of the Himalayan Terai forests of India. International Journal of Conservation Science, 9(4): 749-760.

Bajpai O., Kumar A., Mishra A.K., Sahu N., Pandey J., Behera S.K. \& Chaudhary L.B. (2012). Recongregation of tree species of Katerniaghat Wildlife Sanctuary, Uttar Pradesh, India. Journal of Biodiversity and Environmental Sciences, 2(12): 24-40.

Barua K.N., Gogoi G. \& Hazarika P. (2018). Comparative study on structural composition and community association of Nambor Wildlife Sanctuary and its South-Westward extended Bornewria forest, Assam, India. Tropical Plant Research, 5(2): $233-242$.

Dudley N. (Ed) (2008). Guidelines for applying protected area management categories. Gland, Switzerland, IUCN. Retrieved from: https://portals.iucn.org/library/sites/library/files/documents/PAG-021.pdf

EU (European Union) (2008). Forest biodiversity as a challenge and opportunityfor climate change, adaptation and mitigation. In: Presidency background paper presented at the Informal Meeting of EU Environment Ministers. pp. 12.

Gbile Z.O. (1984). Vernacular names of Nigerian plants Yoruba. Forestry Research Institute of Nigeria, Ibadan, Nigeria.

Husch B., Beers T.W. \& Kershaw J.A.Jr. (2003). Forest mensuration, $4^{\text {th }}, 443$ Hoboken, NJ: John Wiley and Sons, Inc. 
IIRS (Indian Institute of Remote Sensing) (2002). Biodiversity Characterization at Landscape Level in North East, India Using Satellite Remote Sensing and GIS. Indian Institute of Remote Sensing, National Remote Sensing Agency, Dept. of Space, Dehradun 248001, Uttaranchal.

IUCN (1994). Guidelines for Protected Area Management Categories. Gland and Cambridge: IUCN.

Keay R.W.J. (1989). Trees of Nigeria. A revised version of 'Nigeria Trees'. Clarendron Press, Oxford, 476 p.

Kumar A., Marcot B.G. \& Saxena A. (2006). Tree species diversity and distribution patterns in tropical forests of Garo Hills. Current Science, 91: 1370-1381.

Magurran A.E. (2004). Measuring Biological Diversity. Blackwell, Oxford, U.K., pp. 256.

Maradana T.N. \& Owk A.K. (2015). Tree diversity, stand structure and community of tropical forests in Eastern Ghats of Andhra Pradesh, India. Journal of Asia-Pacific Biodiversity, 9: 328-334.

Nurudeen T.A., Aina-oduntan O.A., Awotedu B.F. \& Salami K.D. (2017). Structure and Tree Species Diversity Pattern of Gambari Forest Reserve Southwestern Nigeria. International Journal of Applied Research and Technology, 6(6): 21-29.

Onyekwelu J., Mosandi R. \& Stimm B. (2008). Tree species diversity and soil status of Primary and Degraded Tropical Rainforest Ecosystems in South-Western Nigeria. Journal of Tropical Forest Science, 20(3): 193-204.

Onyekwelu J.C., Mosandl R. \& Stimm B. (2007). Tree species diversity and soil status of two natural forest ecosystem in lowland humid tropical rainforest region of Nigeria. Tropentag 2007. University of Kassel-Witzenhausen and University of Gottingen, October 9-11. Conference of international Agricultural Research Development.

Rao P., Barik S.K., Pandey H.N. \& Tripathi R.S. (1990). Community Composition and tree Population Structure in a Sub-Tropical Broad-leaved Forest along distance gradient. Vegetation 88: 151-162.

Rennolls K. \& Reynolds K.M. (2007). Indicators for biodiversity of Tropical Forest: Problems and solutions. In: Reynolds K.M., Thomson A.J., Kohl M., Shannon M.A., Ray D. \& Rennolls K. (Eds.) Monitoring and Modelling of Knowledge Management and Policy Science. CABI, pp. 103-128.

Sarkar M. \& Devi A. (2017). Analysis of medicinal and economic important plant species of Hollongapar Gibbon wildlife sanctuary, Assam, northeast India. Tropical Plant Research, 4(3): 486-495.

Shi H. \& Singh A. (2002). An assessment of biodiversity hotspots using Remote Sensing and GIS. Journal of the Indian Society of Remote Sensing, 30(1-2): 105-112.

Stohlgren T.J., Binkley D. \& Chong G.W. (1999). Exotic plant species invade hot spots of native plant diversity. Ecology Monograph, 69: 25-46.

Tonolli S., Rodeghiero M., Gianelle D., Dalponte M., Bruzzone L. \& Vescovo L. (2011). Mapping and modeling forest tree volume using forest inventory and airborne laser scanning. European Journal of Forest Research, 130: 569-577.

UNEP-WCMC (2008). State of the World's Protected Areas: An Annual Review of Global Conservation Progress. United Nations Environment Programme World Conservation Monitoring Centre (UNEP-WCMC).

Zheng Z., Feng Z., Cao M., Li Z. \& Zhang J. (2006). Forest Structure and Biomass of a Tropical Seasonal Rainforest in Xishuangbanna, Southwest China. Biotropica, 38: 318-327. 